\title{
AMILOIDOSE E INSUFICIÊNCIA CARDÍACA COM FRAÇÃO DE EJEÇÃO PRESERVADA, UMA ASSOCIAÇÃO MAIS COMUM DO QUE AS DESCRIÇÕES EPIDEMIOLÓGICAS ATUAIS: RELATO DE CASO
}

\author{
AMYLOIDOSIS AND HEART FAILURE WITH PRESERVED EJECTION FRACTION, \\ A MORE COMMOM ASSOCIATION THAN CURRENT EPIDEMIOLOGICAL DESCRIPTIONS: \\ CASE REPORT
}

Mateus Oliveira Potratz' ${ }^{1}$ Caio Guimarães Araujo ${ }^{1}$, Rebeca Silva Moreira da Fraga', Rodolfo Costa Sylvestre ${ }^{2}$, Osmar Araújo Calii ${ }^{3}$

1 Médico Residente do Hospital Universitário Cassiano Antônio Moraes, Vitória, ES - Brasil.

2 Médico Residente do Hospital da Santa Casa de Misericórdia de Vitória, Vitória, ES - Brasil.

3 Professor Adjunto de Cardiologia da Escola de Medicina da Santa Casa de Misericórdia de Vitória, Vitória, ES Brasil.

Instituição na qual o trabalho foi realizado/ está vinculado: Irmandade da Santa Casa de Misericórdia de Vitória: Comitê de Ética em Pesquisa da Escola de Ciências da Saúde-EMESCAM (CEP/EMESCAM), telefone (27) 3334-3586, e-mail comite.eca@emescam.br, endereço: Av. N. S. da Penha, 2190, Santa Luiza - Vitória - ES 29045-402.

Autor correspondente: Mateus Oliveira Potratz, telefone (27)981732085, e-mail: mateusopotratz@ hotmail.com, endereço: R. Sertório Franco, n 97, Sertório Franco, Vitória - ES, CEP 29070835.

\section{Resumo}

A amiloidose é uma doença caracterizada pelo depósito de fibrilas amiloides. Ao se depositarem no coração podem ocasionar distúrbio da condução, cardiomiopatia restritiva, disfunção diastólica, baixo débito e insuficiência cardíaca de fração de ejeção preservada (ICFEP), principalmente relacionada a transtirretina (TTR). Descrição do caso: Homem de 70 anos, hipertenso, diabético e dislipidêmico procurou o cardiologista queixandose de dispneia aos pequenos esforços, hiporexia, adinamia e edema em membros inferiores, com turgência jugular a 45으 e hiperfonese de segunda bulha na ausculta cardíaca. Iniciada a propedêutica complementar para melhor elucidação da etiologia da insuficiência cardíaca com eletrocardiograma em repouso evidenciando baixa voltagem, peptídeo natriurético do tipo B no valor de $3920 \mathrm{pg} / \mathrm{ml}$, ecocardiograma com aumento biatrial, hipertrofia concêntrica do ventrículo esquerdo, função sistólica global do ventrículo esquerdo preservada com fração de ejeção de $65 \%$, septo hipertrófico e dee aspecto infiltrativo heterogêneo e hiperrefringente. Devido à suspeita de amiloidose foi realizada

\begin{abstract}
Amyloidosis is a disease characterized by the deposition of amyloid fibrils. When deposited in the heart, they can cause conduction disturbance, restrictive cardiomyopathy, diastolic dysfunction, low output and preserved ejection fraction heart failure (HFpEF), mainly related to transthyretin (TTR). Case description: A 70-year-old man, hypertensive, diabetic and dyslipidemic sought a cardiologist complaining of dyspnea on mild exertion, hyporexia, adynamia and edema in the lower limbs, with jugular turgescence at $45^{\circ}$ and second heart sound hyperphonesis on cardiac auscultation. Complementary workup started to better elucidate the etiology of heart failure with electrocardiogram at rest showing low voltage, type $B$ natriuretic peptide in the value of $3920 \mathrm{pg} / \mathrm{ml}$, echocardiogram with biatrial enlargement, concentric hypertrophy of the left ventricle, global systolic function of the left ventricle preserved with an ejection fraction of $65 \%$, hypertrophic septum and a heterogeneous and hyperrefringent infiltrative appearance. Due to the suspicion of amyloidosis, an investigation was carried out with cardiac magnetic resonance, which
\end{abstract}


investigação com ressonância magnética cardíaca, que demonstrou realce tardio subendocárdio característico da patologia e posteriormente teste genético para amiloidose de origem familiar associada a variantes no gene da TTR, confirmando o diagnóstico. Conclusão: A amiloidose cardíaca é uma patologia descrita como rara, porém com inúmeros casos subdiagnosticados. Condições antes atribuídas ao envelhecimento normal provavelmente são decorrentes de depósito amiloide cardíaco, levando à ICFEP. A existência de métodos diagnósticos menos invasivos, principalmente com dissociação entre ecocardiograma e eletrocardiograma, corroboram para o diagnóstico precoce, plano de tratamento e melhora do prognóstico, modificando a história natural da doença, evitando a deposição de fibrilas nos tecidos.

Palavras-chave: "Amiloidose", "Cardiomiopatia Restritiva", "Insuficiência Cardíaca".

\section{Introdução}

Amiloidose não é uma doença única, mas todos os espectros dela compartilham uma característica em comum, a deposição extracelular de proteínas patológicas fibrilares em órgãos e tecidos. ${ }^{1}{ }^{2} \mathrm{~A}$ deposição pode alterar a função destes, e quando há acometimento cardíaco, pode levar a disfunção diastólica progressiva e miocardiopatia restritiva. ${ }^{3}$

Devido sua complexidade diagnóstica e de manejo, a amiloidose cardíaca ( $\mathrm{AC}$ ) é muitas vezes subdiagnosticada, ou muitas vezes tardiamente identificada. O diagnóstico definitivo é concedido pela biópsia endomiocárdica, com demonstração do depósito amilóide, utilizando-se técnica de coloração específica.4 Quando a suspeita clínica é alta e não há biópsia, podemos lançar mão de modalidades diagnósticas não invasivas. O tratamento e prognóstico da doença dependem da etiologia e da extensão da deposição amilóide. ${ }^{3}$

Relatamos, a seguir, um caso clínico de um paciente com insuficiência cardíaca com fração de ejeção preservada por deposição de substância amilóide. O diagnóstico foi realizado através de métodos não invasivos, sendo evidenciadas alterações em ressonância nuclear magnética (RNM) características de amiloidose, além de pesquisa genética de mutação do Gene TTR que demonstrated late subendocardial enhancement characteristic of the pathology, and later a genetic test for amyloidosis of familial origin associated with variants in the TTR gene, confirming the diagnosis. Conclusion: Cardiac amyloidosis is a condition described as rare, but with numerous underdiagnosed cases. Conditions previously attributed to normal aging are likely due to cardiac amyloid deposits, leading to HFpEF. The existence of less invasive diagnostic methods, especially with dissociation between echocardiogram and electrocardiogram, contribute to early diagnosis, treatment plan and improved prognosis, modifying the natural history of the disease, avoiding the deposition of fibrils in the tissues.

Key Words: "Amyloidosis", "Cardiomyopathy, Restrictive", "Heart Failure"

confirmou amiloidose hereditária ligada á Transtirretina.

\section{Relato de Caso}

Homem de 70 anos, hipertenso, diabético e dislipidêmico, sem histórico de insuficiência cardíaca, em uso domiciliar de Losartana $50 \mathrm{mg}$ de 12/12 horas, Metformina 500 mg no café da manhã, almoço e jantar e Sinvastatina $20 \mathrm{mg}$ à noite, procurou assistência médica em cardiologista há 01 ano, queixando-se de dispneia aos pequenos e médios esforços, hiporexia, adinamia e edema em membros inferiores, com turgência jugular a 450 e hiperfonese de segunda bulha na ausculta cardíaca. Iniciada a propedêutica complementar para melhor elucidação diagnóstica e da etiologia da insuficiência cardíaca. Eletrocardiograma em repouso demonstrou baixa voltagem em derivações uni e bipolares (Figura 01); peptídeo natriurético do tipo $B$ com valor de $3920 \mathrm{pg} / \mathrm{ml}$; ecocardiograma transtorácico com aumento biatrial, hipertrofia concêntrica do ventrículo esquerdo, função sistólica global do ventrículo esquerdo preservada com fração de ejeção de $65 \%$, septo interventricular de 15 $\mathrm{mm}$ e aspecto infiltrativo heterogêneo e hiperrefringente (Figura 02), pressão sistólica da artéria pulmonar de $57 \mathrm{mmHg}$; angiotomografia pulmonar sem alterações significativas; e 
cintilografia miocárdica sem sinais de isquemia. Devido à forte suspeita de amiloidose foi realizada investigação com ressonância magnética cardíaca (Figuras 03, 04 e 05), que evidenciou realce tardio subendocárdio proeminente característico da patologia e posteriormente teste genético para amiloidose de origem familiar associada a variantes no gene da transtirretina, confirmando o diagnóstico.

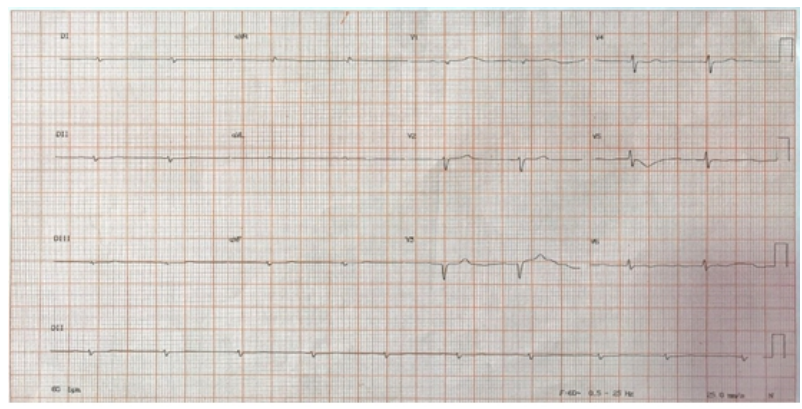

Figura 1: Eletrocardiograma Amiloidose

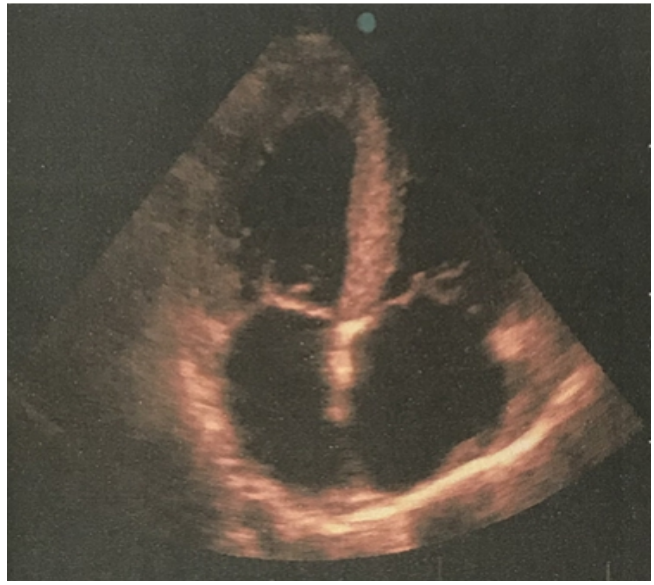

Figura 2: Eco com Hiperrefringência

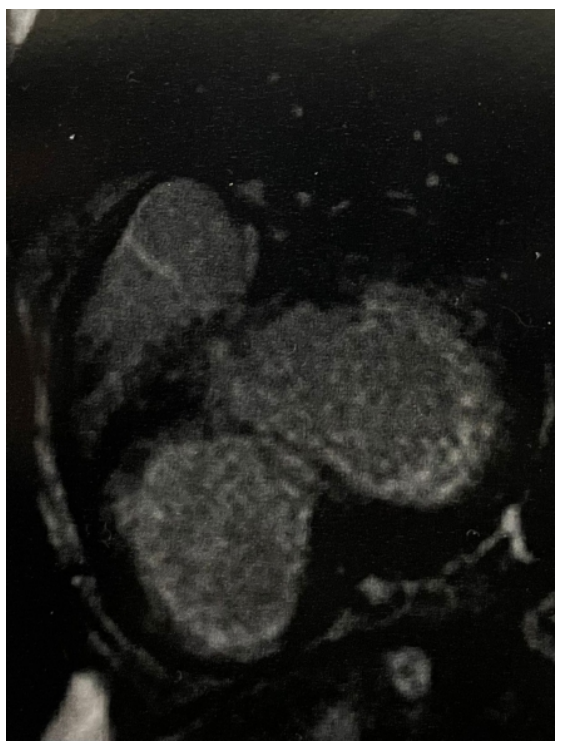

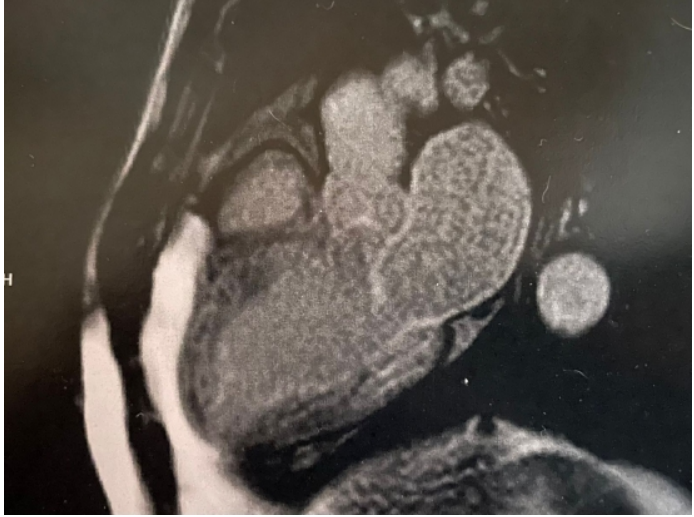

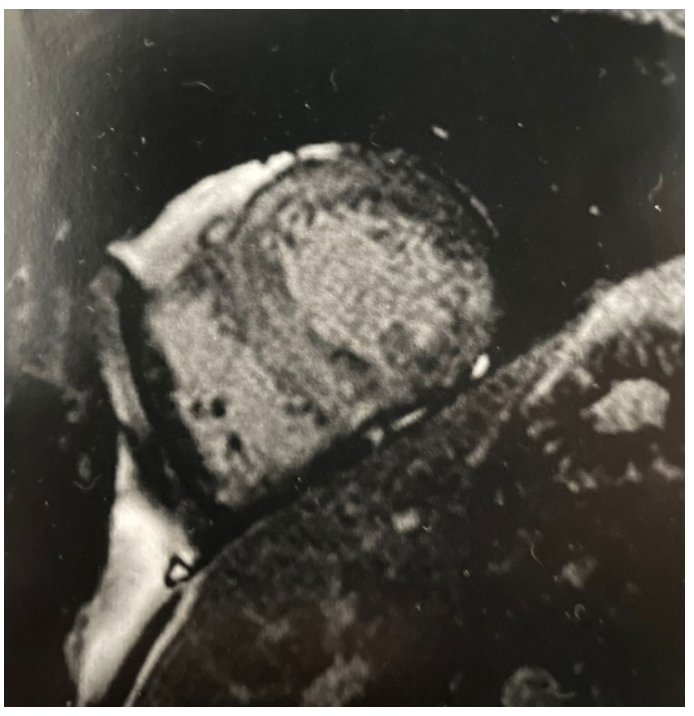

Figura 3, 4 e 5: Ressonância Magnética Cardíaca (RMC)

\section{Discussão}

A amiloidose caracteriza-se pela deposição localizada ou sistêmica de proteínas com estrutura terciária instável, que se agregam e formam as fibras. Estas se deposita em diversos órgãos como rins, pulmões, fígado e até em partes moles. Estes depósitos podem resultar em disfunção do órgão ou tecido afetado. ${ }^{5}$

O quadro clínico da AC é bastante variável e pode ser manifestar como insuficiência cardíaca associada ao aumento da espessura miocárdica, derrame pericárdico, bloqueio atrioventricular, aumento da espessura do septo interventricular e/ou valvular, alterações de deformidade com preservação apical ao ecocardiograma; história de síndrome do túnel do carpo bilateral (especialmente em homens), ruptura atraumática do tendão do bíceps, dor neuropática sem explicação, hipotensão ortostática e diagnóstico de hipertrofia ventricular sem causa aparente. $.^{5}, 6$ 
A AC é uma cardiopatia frequentemente subdiagnosticada e uma causa importante de insuficiência cardíaca. Existem vários tipos de proteínas amiloides conhecidas, mas somente algumas infiltram o coração, causando cardiopatia. São elas: Imunoglobulina de Cadeia Leve (AL), imunoglobulina de cadeia pesada, Transtirretina (TTR), amiloide sérica A e apolipoproteína Al. A forma TTR é a mais comum, seguida da AL. ${ }^{7}$

A amiloidose ATTR é causada pela proteína transterretina. A mesma é produzida pelo fígado e circula na forma de um homotetrâmero, agindo como transportador para tiroxina e como proteína de ligação do retinol. No entanto, a desestabilização da proteína TTR promove a dissociação em monômeros, que adquirem a forma fibrilar e se depositam nos tecidos. ${ }^{8}$

São dois os tipos de transtirretina que vão se depositar de forma sistêmica. O tipo selvagem, tradicionalmente chamado de "amiloidose sistêmica senil", devido ao aumento de sua prevalência conforme a idade. E o tipo variante ou hereditário, chamado de polineuropatia amiloide familiar. Este está relacionado a mais de cem mutações gênicas. $O$ alelo mais comum nos Estados Unidos, causado por uma substituição de valina por isoleucina na posição 122 (Val122lle), é encontrado em $3,4 \%$ dos afro-americanos. ${ }^{9}{ }^{13}$.

O padrão de envolvimento miocárdico na amiloidose ATTR difere da amiloidose AL e varia de acordo com a mutação específica da transtirretina responsável pela doença. Na variante Met 30 da transtirretina, a mutação mais comum, o ecocardiograma geralmente é normal, embora o eletrocardiograma pode mostrar evidências de doença de condução na forma de disfunção do nó sinusal, bloqueio de ramo ou bloqueio atrioventricular. Pacientes com a variante da transtirretina Ala 60 e várias outras mutações têm infiltração miocárdica que é indistinguível do causado por amiloidose AL na ecocardiografia. No entanto, disfunção cardíaca é menos comum e o prognóstico é melhor do que para pacientes com doença cardíaca e amiloidose AL apesar da semelhança dos achados ecocardiográficos. ${ }^{1}$

O diagnóstico de amiloidose é baseado em um quadro clínico suspeito, apoiado por exames de imagem e estabelecido por biópsia tecidual. 0 depósito de amiloide tipicamente leva ao engrossamento das paredes ventriculares, redução da distensibilidade e consequente disfunção diastólica grave, com manifestação de sintomas de insuficiência cardíaca. O quadro clínico de insuficiência cardíaca (IC), como o do nosso paciente relatado, é a apresentação mais comum. ${ }^{14},{ }^{15}$ No entanto, arritmias graves, angina e síncope também podem ocorrer, além de sintomas inespecíficos como fadiga, perda de peso, astenia, diarreia ou constipação devido a disfunção autonômica. ${ }^{3}$

- Dentre os exames complementares, a presença de baixa voltagem no eletrocardiograma (amplitude QRS $<0,5 \mathrm{mV}$ em todas as derivações dos membros ou $<1,0 \mathrm{mV}$ em todas as derivações precordiais) é comum, assim como espessamento da parede ventricular e evidência de disfunção diastólica no ecocardiograma. A ressonância também contribui para o diagnóstico precoce, quando mesmo em estágio iniciais pode identificar depósito subendocárdico difuso. ${ }^{14}$

A biópsia de um órgão envolvido confirmará o diagnóstico. Quando a suspeita clínica é alta e há dificuldade de sítios para biópsia, o procedimento mais simples é obter uma amostra de gordura abdominal subcutânea. Um aspirado de gordura corado com vermelho do Congo será positivo em $85 \%$ dos pacientes com amiloidose AL. ${ }^{16},{ }^{17}$ A biópsia endomiocárdica está indicada quando não se consegue comprovação por amostras extracardíacas ou quando só houver acometimento cardíaco. ${ }^{15}$

O quadro clínico de IC, associado aos achados de baixa voltagem no eletrocradiograma, fração de ejeção preservada, infiltrado de aspecto granulado no ecocardiograma e ressonância com infiltrado subendocárdico, nos possibilitou chegar ao provável diagnóstico de AC. A confirmação da mutação do Gene TTR para amiloidose hereditária corroborou fortemente esse diagnóstico e possibilitou tratamento de suporte adequado.

O prognóstico na amiloidose do ATTR varia com a mutação específica e o tempo de diagnóstico. Em geral, mutações de transtirretina associadas a idade mais jovem no início da doença podem predizer neuropatia progredindo mais rapidamente e cardiomiopatia. ${ }^{1}$

O tratamento da AC tem como objetivos o suporte clínico com controle dos sintomas da insuficiência cardíaca e/ou arritmias, e o atraso da deposição de amiloide através de tratamento específico. ${ }^{15} \mathrm{Na}$ AC por ATTR, até alguns anos atrás, o tratamento era basicamente o transplante 
hepático. ${ }^{18}{ }^{20},{ }^{13} \mathrm{Em}$ alguns pacientes com envolvimento cardíaco sintomático, transplante combinado de fígado e coração era aventado. ${ }^{13}$

Mais recentemente novas estratégias terapêuticas surgiram com objetivo de reduzir a produção de TTR, ou estabilizar a TTR, ou eliminar as fibrilas já depositadas, dentre elas tem-se o Tafamidis e o Diflunisal, que trouxeram grande impacto para o tratamento principalmente do tipo variante, mas também do selvagem. O Tafamidis mostrou poder reduzir progressão tanto da polineuropatia quanto da cardiomiopatia amilóide ATTR. ${ }^{21}{ }^{23}$

\section{Conclusão}

A amiloidose é uma patologia rara, onde o acometimento de múltiplos órgãos e tecidos pode ocorrer. O envolvimento cardíaco é marcador de pior prognóstico, sendo imprescindível o levantamento desta condição como hipótese diagnóstica nos pacientes que se apresentam com miocardiopatia restritiva, principalmente naqueles com mais de 50 anos e sem etiologia definida.

$\mathrm{O}$ rápido reconhecimento da amiloidose é de fundamental importância, visto que a instituição de tratamento precoce pode modificar a história natural da doença, evitando a deposição de novas fibrilas amilóides nos tecidos. Portanto, é importante auxiliarmos no reconhecimento, diagnóstico e instituição de tratamento adequado para esta doença, visando a redução da morbimortalidade nesses cenários.

\section{Referencias}

1. Falk RH, Comenzo RL, Skinner M. The systemic amyloidoses. N Engl J Med. 1997 Sep 25;337(13):898-909.

2. Xavier FC. Amiloidose: revisão bibliográfica [dissertação]. Portugal: Universidade da Beira Interior; 2008.

3. Vieira TA et al. Cardiac Amyloidosis: Restrictive Cardiomyopathy Prototype and Diastolic Dysfunction - Case Report. Arq Bras Cardiol: Imagem cardiovasc. 2017;30(1):13-17.

4. López-Sainz Á, Hernandez-Hernandez A, Gonzalez-Lopez E, Domínguez F, Restrepo-Cordoba MA, CoboMarcos M, Gómez-Bueno M, Hernandez-Perez FJ, Oteo JF, Mirelis JG, Cavero MA, Moñivas V, Mingo Santos S, de Haro-Del Moral FJ, Krsnik I, Salas C, Bornstein B, Briceño A, López JA, Vázquez J, Alonso-Pulpón L, Segovia J, Garcia-Pavia P. Clinical profile and outcome of cardiac amyloidosis in a Spanish referral center. Rev Esp Cardiol (Engl Ed). 2021 Feb;74(2):149-158. English, Spanish.

5. Maurer MS, Elliott P, Comenzo R, Semigran M, Rapezzi C. Addressing common questions encountered in the diagnosis and management of cardiac amyloidosis. Circulation.2017;135(14):1357-77.

6. Milani P, Merlini G, Palladini G. Light chain amyloidosis. Mediterr J Hematolog Infect Dis. 2018;10(1):e2018022.

7. Rahman T, Agarwal VV and Reiss CK. Cardiac amyloidosis: a case series of 5 patients, diagnostic challenges and recent advancement in the treatment of attr amyloidosis. J Am Coll Cardiol. 2020 Mar, 75 (11_Supplement_1) 2898

8. Comenzo R, Zhang Y, Martinez C, Osman K, Herrera G. The tropism of organ involvement in primary systemic amyloidosis: contributions of Ig V (L) germ line gene use and clonal plasma cell burden. Blood. 2001;98 (3):71420.

9. Ton VK, Mukherjee M, Judge DP. Transthyretin cardiac amyloidosis: pathogenesis, treatments, and emerging role in heart failure with preserved ejection fraction. Clin Med Insights Cardiol. 2014;8(Suppl 1):39-44.

10. Yamashita T, Hamidi A K, Yazaki M, Benson MD. A prospective evaluation of the transthyretin lle122 allele frequency in an African-American population. Amyloid. 2005;12(2):127-30.

11. Connors LH, Lim A, Prokaeva T, Roskens VA, Costello CE. Tabulation of human transthyretin (TTR) variants, 2003. Amyloid. 2003;10(3):160-84.

12. Jacobson DR, Pastore RD, Yaghoubian R, Kane I, Gallo G, Buck FS, et al. Variant-sequence transthyretin (isoleucine 122) in late-onset cardiac amyloidosis in black Americans. N Engl J Med. 1997;336(7):466-73.

13. Koike H, Katsuno M. Ultrastructure in Transthyretin Amyloidosis: From Pathophysiology to Therapeutic Insights. Biomedicines. 2019 Feb 5;7(1):11. doi: 10.3390/biomedicines7010011.

14. Tovar Echeverría, M., Auquilla Clavijo, P.E., Escobedo Palau, J.A., Navarro Beltrán, P., \& Povar Marco, J.. (2018). Diagnóstico no invasivo de amiloidosis cardiaca por transtirretina. Caso clínico. 
15. E.Gonzalez-Lopez et al. Diagnostico y tratamento de la amiloidosis cardíaca por transtiretina. Progreso y esperanza. Rev Esp Cardiol. 2017; 70(11):991-1004.

16. Libbey CA, Skinner M, Cohen AS. Use of abdominal fat tissue aspirate in the diagnosis of systemic amyloidosis. Arch Intern Med 1983;143:1549- 52.

17. Duston MA, Skinner M, Shirahama T, Cohen AS. Diagnosis of amyloidosis by abdominal fat aspiration: analysis of four years' experience. Am J Med 1987;82:412-4.

18. Holmgren G, Ericzon B-G, Groth C-G, et al. Clinical improvement and amyloid regression after liver transplantation in hereditary transthyretin amyloidosis. Lancet 1993;341:1113-6.

19. Skinner $M$, Lewis WD, Jones LA, et al. Liver transplantation as a treatment for familial amyloidotic polyneuropathy. Ann Intern Med 1994;120: 133-4.

20. Steen L, Holmgren G, Suhr O, Wikstrom L, Ericzon B-G, Groth C-G. World-wide survey of liver transplantations in patients with familial amyloidotic polyneuropathy. Amyloid Int J Exp Clin Invest 1994;1:138-42.

21. Coelho T, Merlini G, Bulawa CE, Fleming JA, Judge DP, Kelly JW, Maurer MS, Planté-Bordeneuve V, Labaudinière R, Mundayat R, Riley S, Lombardo I, Huertas P. Mechanism of Action and Clinical Application of Tafamidis in Hereditary Transthyretin Amyloidosis. Neurol Ther. 2016 Jun;5(1):1-25. 22. Maurer MS, Grogan DR, Judge DP, ET al. Tafamidis in transthyretin amyloid cardiomyopathy: effects on transthyretin stabilization and clinical outcomes. Circ Heart Fail 2015; 8: 519-26.

23. Maurer MS, Schwartz JH, Gundapaneni B, Elliott PM, Merlini G, Waddington-Cruz M, Kristen AV, Grogan M, Witteles R, Damy T, Drachman BM, Shah SJ, Hanna M, Judge DP, Barsdorf Al, Huber P, Patterson TA, Riley S, Schumacher J, Stewart M, Sultan MB, Rapezzi C; ATTR-ACT Study Investigators. Tafamidis Treatment for Patients with Transthyretin Amyloid Cardiomyopathy. N Engl J Med. 2018 Sep 13;379(11):1007-1016. 